\title{
SUPPORTING DATA INTENSIVE APPI.ICATIONS WITH MEDIUM GRAINED PARALLELISM
}

July 1, 1991 - February 28, 1992

DOE/ER/25063--T13

Progress Report

DE92 015101

Submitted to:

Environmental Acquisitions Branch

Procurement and Contracts Division

U. S. Department of Energy

Oak Ridge Operations

Federal Building

200 Administration Road

Oak Ridge, TN $37831-8758$

Attention:

Ms. Barbara J. Jackson, AD-423

Contract Management Branch

Submitted by:

John L. Pfaltz, Professor

James C. French, Research Assistant Professor

Andrew S. Grimshaw, Assistant Professor

Sang H. Son, Assistant Professor

SEAS Report No. UVA/527435/CS92/101

April 1992

\section{PREPARED FOR THE U. S. DEPARTMENT OF ENERGY}

UNDER GRANT NUMBER DE-FG05-88ER25063

\section{DEPARTMENT OF COMPUTER SCIENCE DISCLAIMER}

\begin{abstract}
This report was prepared as an account of work sponsored by an agency of the United States Government. Neither the United States Goveinment nor any agency thereof, nor any of their employees, makes any warranty, express or implied, or assumes any legal liability or responsibility for the accuracy, completeness, or usefulness of any information, apparatus, product, or process disclosed, or represents that its use would not infringe privately owned rights. Reference herein to any specific commercial product, process, or service by trade name, trademark, manufacturer, or otherwise does not necessarily constitute or imply its endorsement, recommendation, or favoring by the United States Government or any agency thereof. The views and opinions of authors expressed herein do not necessarily state or reflect those of the United States Government or any agency thereof.
\end{abstract}




\begin{abstract}
A Progress Report
Grant No. DE-FG05-88ER25063

July 1, 1991 - February 28, 1992 MEDIUM GRAINED PARALLELISM
\end{abstract}

SUPPORTING DATA INTENSIVE APPLICATIONS WITH

\author{
Submitted to: \\ Environmental Acquisitions Branch \\ Procurement and Contracts Division \\ U. S. Department of Energy \\ Oak Ridge Operations \\ Federal Building \\ 200 Administration Road \\ Oak Ridge, TN 37831-8758
}

Attention:

Ms. Barbara J. Jackson, AD-423

Contract Management Branch

Submitted by:

John L. Pfaltz, Professor

James C. French, Research Assistant Professor:

Andrew S. Grimshaw, Assistant Professor

Sang H. Son, Assistant Professor

Department of Computer Science

SCHOOL OF ENGINEERING AND APPLIED SCIENCE

UNIVERSITY OF VIRGINIA

CHARLOTTESVILLE, VIRGINIA

Report No. UVA/527435/CS92/101

Copy No.

April 1992 


\section{Table of Contents}

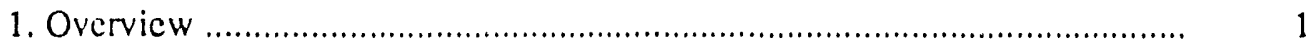

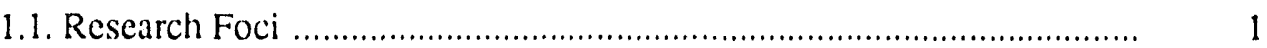

1.2. Niajor Achievements .................................................................... 2

1.3. Publications ................................................................................ 2

Book Chapters ......................................................................... 2

Journal articles ............................................................................ 3

Conference procededings .............................................................. 3

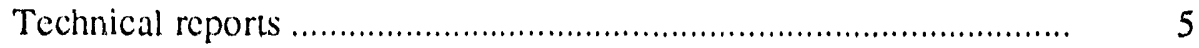

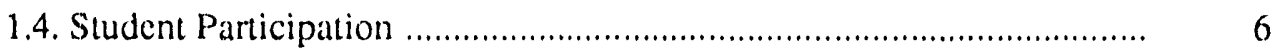

2. Parallelization of Database Constructs and Operators .................................. 8

2.1. Extensible File System ..................................................................... 8

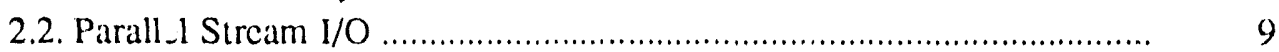

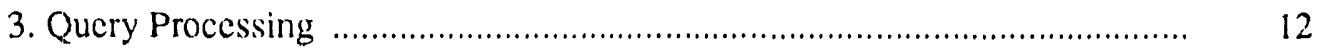

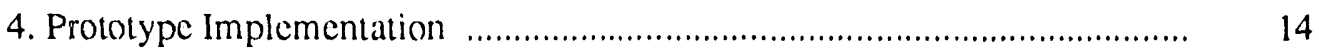

5. Synchronization and Reliability: Algorithms and New Paradigms ............. 15

6. Spatial Databases ........................................................................... 16

7. Dynamic Schema Modification and Metadata .......................................... 17

7.1. Shared Dictionary Development ........................................................... 17

7.2. Representation of Scientific Data ........................................................ 18

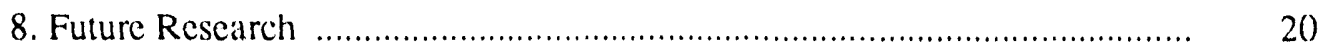

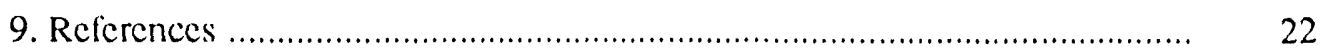




\section{Overview}

ADAMS $^{1}$ is an ambitious effort to provide new database access paradigms for the kinds of scientific applications that require massively parallel access to very large data sets in order to be effective. Many of the Grand Challenge Problems fall into this category, as well as those kinds of scientitic research which depend on widely distributed, shared sets of disparate data.

The essence of the ADAMS approach is to view data purely in functional terms, rather than the more traditional structural view in which multiple data items are aggregated into records or tuples of flat files [PFG92, Pfa92]. Further, ADAMS has been implemented as an embedded interface so that scientists can develop applications in the host programming language of their choice, often Fortran, Pascal, or C, and still access shared data generated in other environments. The syntax and semantics of ADAMS is essentially complete. ${ }^{2}$ The functional nature of the ADAMS data interface paradigm simplifies its implementation in a distributed environment, e.g. the Mentat run-time system [Gri90], because one must only distribute functional servers, not pieces of data structures. However, this only opens up the possibiliiy of effective parallel database processing; to realize this potential far more work must be done in the areas of data dependence, intra-statement parallelism [GPF9()], parallel query optimization, and maintaining consistency and reliability in concurrent systems [SSC91, SoC91]. Discovering how to make effective parallel data access an actuality in real scientific applications is the point of this research.

\subsection{Research Foci}

In Section 4 of our 3 year grant proposal, we outlined three major areas of research to be pursued during the grant period. They were:

4.1.1 Parallelization of Database Constructs and Operators.

Part (1), "Explore the effect of object granularity on parallelizition and throughput in intra-statement parallelism" is discussed in Section 2. Parts (2) and (3) which are more complex are under current invest 'gation.

4.1.2 Parallel Synchronization and Concurrency Protocols.

Part (1), "Model concepts of trinsaction correctness different from serializability" and part (2) "Investigate the effects of performance of communication cost and topology for a set of selected concurrency control protocols" are discussed in Section 5. We have obtaineci several excellent preliminary results.

4.1.3 Query Processing

Part (1) "Model possible vertical yuery decomposition., given a $1^{\text {st }}$ order predicate set characterization" is described in Section 3. It extension to full parallel query capability is under development.

In the same section, we noted that considerable development work still remained before we would have a fully functional implementation. They were:

\footnotetext{
${ }^{1}$ Advanced DAta Management System.

${ }^{2}$ [PFG91] provides a preliminary introduction, and we have included current (unpublished) working descriptions of both the syntax and semantics in this report.
} 


\subsubsection{Generating Parallel Code.}

No substantial progress on either of these was made in this period. The code generator was rewritten to clean up a number of gross inefficiencies, but it has not yet been extended to automatically generate the kinds of parallel code explored in Sertion 2. The storage manager is in the proces $s$ of being rewritten to run on the Intel iPSC-2 hypercube under their concurrent file system.

\subsubsection{Hierarchical. Name Spaces.}

Active investigation of part (1), "Investigate the benefits of generalizing the dictionary to accommodate an increased, but fixed number of scope levels", has been deferred until later in the grant period. However, we have begun to rewrite the dictionary interface to accommodate it, as described in Section 7. This work will also be the basis of part (3) "Implement dynamic dictionary maintenance", which has largely been accomplished.

We have cited the actual paragraphs of our grant proposal because we are using it as a road map in planning and scheduling our on-going research. However, as is true will all real research, we have encountered a number of interesting phenomena which were unanticipated when we originally proposed this grant. Since these discoveries have arisen directly from the research of this grant, we have described them in Sections 6 and 7.

\subsection{Major Achievements}

We are quite proud of the overall research and development results that we have obtained during the grant period; but we recognize that some are only incremental, while others we would categorize as major achievements. The ones that we consider to be in the latter category are:

(1) the design and implementation of extensible file systems that provide the access information needed for optimization on a class-by-class basis, described in Section 2 ;

(2) the inplementation of parallel stream $\mathrm{I} / \mathrm{O}$, described in Section 2;

(3) the development of new scheduling algorithms based on the idea of dynamically adjusting the serialization order of active transactions, in Section 5; and

(4) the use of dynamic schema modification in database design, described in Section 7.

\subsection{Publications}

The work conducted on this project in the last 8 months has led to the following list of technical reports, conference proceedings, and journal articles. Copies of each have been included in this report. More are in preparation.

\section{Book Chapters}

S. H. Son, Y. Lin, and R. Cook, "Concurrency Control in Real-Time Database Systems" in Foundations of Real-Time Computing: Scheduling and Resource Management, A. Van Tilborg and G. M. Koob (Edilors), Kluwer Academic Publishers, 1991, 
pp 185-2()2.

J. L. Pfaltz, "Forward", in Introduction to Object-Oriented Databases, by D.N. Chorafas, and H. Steinmann, Prentice-Hall, 1992 (to appear).

S. H. Son, R. Cook, J. Lec, and H. Oh, "New Paradigms for Real-Time Database Systems" in Real-Time Programming, K. Ramamritham and W. Halang (Editors), Pergamon Press, 1992 (to appear).

S. H. Son and S. Park, "Scheduling Transactions for Distributed Time-Critical Applications" in Advances in Distributed Computing: Concepts and Design, T. Casavant and M. Singhal (Editors), IEEE Computer Socicty Press, 1992 (to appear).

\section{Journal Articles:}

P. Shebalin, S. H. Son, and C. Chang, "An Approach to Software Safety Analysis in Distributed Systems", Computer Syistems Science and Engineering, vol. 6, no. 2, April 1991, pp 102-116.

L. Sha, R. Rajkumar, S. H. Son, and C. Chang, "A Real-Time Locking Protocol" IEEE Transactions on Computers, vol. 40, no. 7, July 1991, pp 793-800.

S. H. Son, "An Environment for Integrated Development and Evaluation of Real-Time Distributed Database Systems", Journal of Systems Integration, vol. 2, no. 1, Feb. 1992, pp 67-90.

S. H. Son, J. Ratner, S. Chiang, "StarBase: A Simulation Laboratory for Distributed Database Research", Journal of Computer Simulation. (10 appear).

J. C. French, T. W. Pratt, M. Das, "Performance Measurement of the Concurrent File System of the Intel iPSC/2 Hypercube", Journal of Parallel and Distributed Computing, (submilled).

\section{Conference Proceedings:}

A. S. Grimshaw, V. E. Vivas, "FALCON: A Distributed Scheduler for MIMD Architectures", Proceedings of the Symposium on Experiences with Distributed and Multiprocessor Systems, Allanta, GA, March, 1991.

S. H. Son, P. Wagle, and S. Park, "Real-Time Database Scheduling: Design, Implementation, and Performance Evaluation", The Second International Symposium on Database Systems for Advanced Applications (DASFAA '91), Tokyo, Japan, April 1991, pp 146-155.

H. Kang and S. H. Son, "A Hicrarchical Export/Import Scheme for Data Sharing in a Federated Distributed Databalse System," The Second International Symposium on Database Systems for Advanced Applications (DASFAA '91), Tokyo, Japan, April 1991, pp 31-40.

A. S. Grimshaw, J. Prem, "High Performance Parallel File Objects", Proceedings of the Sixth Distributea Memory Computing Conference, Pontland, OR., April 1991. 
S. H. Son, C. Iannacone, and R. Beckinger, "Integrating Databases with Real-Time Computing Systems," IEEE Southecatcon '9l. Williamsburg, Virginia, A pril 1991, pp 837-841.

R. P. Cook, S. H. Son, H. Y. Oh, and J. Lee, "New Paradigms for Real-Time Database Systems," 8th IEEE Workshop on Real-Time Operating Systems and Software, Atlanta, Georgia, May 1991, pp 103-108.

S. H. Son, C. Iannacone, and M. Poris, "RTDB: A Real-Time Database Manager for Time-Critical Applications," Euromicro Workshop on Real-Time Systems, Paris, France, June 1991, pp 207-214.

A. S. Grimshaw, "A Software Environment for High-Performance Parallel Computing", Proceedings 1991 Minnowbrook Workshop on Software Engineering for Parallel Computing, July 1991.

S. H. Son and S. Chiang, "Evaluation of a Concurrent Checkpointing Algorithm for Distributed Database Systems," International Conference on Parallel and Distributed Computing and Systems. Washington, DC, October 1991, pp 125-129.

A. S. Grimshaw, E. C. Loyot Jr., "ElFS: Object-Oriented Extensible File Systems", Proc. 1991 Parallel and Distrihuted Information Systems Conference, Miami, FL, December 1991.

Y. Oh and S. H. Son, "Multiprocessor Support for Real-Time Fault-Tolerant Scheduling," IEEE Workshop on Architectural Aspects of Real-Time Systems, San Antonio, Texas, December 1991, pp 76-80.

J. L. Pfaltz, J. C. French, A. S. Grimshaw, R. D. McElrath, "Functional Data Representation in Scientific Information Systems", Inter. Space Year Conf. on Earth and Space Science Information Systems, Pasadena, CA, Feb. 1992.

J. C. French, "An Environment for Passively Sharing Inlormation", Inter. Space Year Conf. on Earth and Space Science Information Systems, Pasadena, CA, Feb. 1992.

S. H. Son, J. Lee, and S. Shamsunder, "Real-Time Transaction Processing: Pessimistic, Optimistic, and Hybrid Approaches," Second International Workshop on Transactions and Query Processing. Tempe, Arizona, February 1992.

S. H. Son, S. Park, and Y. Lin, "An Integrated Real-Time Locking Protocol," Eighth IEEE International Conference on Data Engineering, Phoenix, Arizona, Fcbruary 1992, pp 527-534.

R. Orlandic, J. L. Pfalli, "A Highly Compressed B-tree Index for Long, Variable-Length Strings", Proceedings DCC'92 Conft: Snowbird, Utah, March 1992, (to appear).

R. Orlandic, J. L. Pfaltz, "Retrieving Dalla Based on Long Keys", Proceedings" of the Navy Environmental Systems Workshop. Snowbird, Utah, March 1992, (to appear). 
J. L. Pfaltz, "A Functional Approach to Scientific Database Implementation", $\sigma^{\text {th }}$ International Working Conf. on Scientific and Statistical Database Management, Zurich, Switzerland, June 1992, (to appear).

R. Orlandic, "New Quadtrec Representation of Spatial Images and Its Application for Spatial Scarch in Geographic Databases", 6th SSDBM CONF on Scientific and Statistical Database Management Switzerland, June 1992, (10 appear).

S. H. Son and J. Lee, "A New Approach to Real-Time Transaction Scheduling," 4th Euromicro Workshop on Real-Time Syistems, Athens, Grecce, June 1992 (to appear).

Y. Oh and S. H. Son, "An Algorithm for Real-Time Fault-Tolerant Scheduling in Multiprocessor Systems," 4th Euromicro Workishop on Real-Time Systems Athens, Grece, Junc 1992 (10 appear).

S. H. Son, S. Yannopoulos, Y-K. Kim, C. Iannacone, "Integration of a Database System with Real-Time Kemel for Time Critical Applications," Second International Conference on System Integration. Morristown, New Jersey, June 1992 (to appear).

S. H. Son and S. Koloumbis, "Replication Control for Distributed Real-Time Database Systems," 12th International Conference on Distributed Computing Systems, Yokohama, Japan, June 1992 (10 appear).

J. L. Pfaltz, J. C. Trench, "Multiple Inheritance and the Closure oi Set Operators in Class Hicrarchies", International Conf. on Data Theory, '92, Berlin, Germany, Oct. 1992, (submitted).

\section{Technical Reports:}

J. L. Pfaltz, J. C. French, A. Grimshaw, "An Introduction to the ADAMS Interface Language: Part i" IPC TR-9/-()6. Institute for Pallallel Computation, Univ. of Virginia April 1991.

S. H. Son and S. Koloumbis, "Performance Evaluation of Replicated Control Algorithms for Distributed Database Systems," Technical Report TR-91-1/. Dept. of Computer Science, University of Virginia, May 1991.

S. H. Son and R. Beckinger, "MRDB: A Multi-User Real-Time Dalabase Manager for Time-Critical Applications," Technical Report TR-9/-13, Dept. of Computer Science, University of Virginia, May 1991.

A. S. Grimshaw and E. C. Loyol, Jr., "ELFS: Object-Oriented Extensible File Systems", Technical Report TR-91-14 Dept. of Compuler Science, University of Virginia, July 1991.

T. P. Clcary, "A Relational Interface to an Object Based System, or Translating SQL to ADAMS" IPC TR-9/-0)9 Institute "or Parallel Computation, Univ. of Virginia Aug. 1991. 
R. Orlandic, J. L. Pfaltz, "Q,-trees: A Dynamic Structure for Accessing Spatial Objects with Arbitrary Shapes", IPC TR-9/-()/0 Institute for Parallel Computation, Univ. of Virginia Dec. 1991.

R. McElrath, "A Look at Two Persistent Storige Models", IPC TR-9/-01/ Institute for Parallel Computation, Univ. of Virginia, Dec. 1991.

Y. Yang, L. Hsu, and S. H. Son, "Distributed Algorithms for Efficient Deadlock Detection and Resolution," Technical Report TR-92-06. Dept. of Computer Science, University of Virginia, February 1992.

\subsection{Student Participation}

In addition to the the principal investigators, we have attracted considerable student interest, allowing us to select only those who we believe to be the most productive. Many of these are Ph.D. candidates who expect to complete their dissertations in database research. Below we have listed the 19 students who have been active in this project, together with their expected degrees and primary area of contribution. It should be noted that only a few of these students have been directly supported under this grant. Some are self-supporting, others have received very minimal support to augment other sources; but the research of all has been relevant to and has, in some waly, been facilitated by this grant.

\section{Participating Students:}

- David Baker (M.S. awarded December 1991), distributed transaction processing

- Robert Beckinger (M.S. awarded May 1991), multi-user temporal database

- Shi-Chin Chiang (Ph.D.), database checkpointing methods

- Gokul Das (M.S. awarded December 1991), index tree in multiprocessor databases

- Mriganka Das (Ph.D.), clustered retrieval

- Lana Durant (M.S.), document representation and retrieval

- Russell Haddleton (Ph.D.), parallel storage management

- Carmen Iannacone (M.S. awarded May 1991), imprecise database server

- Young-Kuk Kim (Ph.D.), OS kernel support for database manager

- Spiros Koloumbis (M.S. awarded May 1991), replication contro algorithms

- Juhnyoung Lee (Ph.D.), database scheduling and concurrency control

- Lindsay Loyd (M.S., awarded Aug. 1991), implementation of O-tree access, array representation

- Edmond Loyot (Ph.D.), extensible file systemis

- Rodney McElrath (M.S., awarded Jan. 1992), run-time system

- Henry Oh (Ph.D.), fault-tolerant scheduling in multiprocessor systems

- Savita Shamsunder (M.S.), optimistic concurrency control

- Stavros Yannopolous (M.S.), real-time datibase server 
- Fengjie Zhang (M.S. awarded December 1991), replication control evaluation - Weifeng Zheng (Ph.D.) predicates and semantic databases 


\section{Parallelization of Database Constructs and Operators}

Although, in our original proposal we thought it would be sufficis at to obtain high performance through simply establishing parallel threads of execution within individual ADAMS statements (intra-statement parallelism), and then later between separate ADAMS statements (inter-statement parallelism) we found that this by itself would not be sufficient. A more effective, very low-level parallelism is also required.

\subsection{Extensible File System}

The problem is that contemporary high performance computer systems have become increasingly unbalanced. CPU speeds have increased dramatically over the last decade. At the same time $1 / O$ performance has improved only marginally. Thus, the performance of many scientific applications is bound by the performance of the I/O system. The advent of highly parallel architectures has made the problem even worse. For example, the Intel 128 node $\mathrm{PSSC} / 860$ ) has a peak performance of 7680 double precision mega-flops. Yet IO latency is still in the 10-20 millisecond range, and the aggregate bandwidth is only on the order of $2 \mathrm{MB} / \mathrm{sec}$ ond for a 4 node $\mathrm{IO}$ system. What this means for scientific programmers is that their applications will be more $1 / O$ bound than ever before on the new machines, and they will be unable to fully exploit these new architectures to solve ever larger problems.

Parallel disk arrays [PGK88, PiP89] and disk striping [SaG86] are seen by many as the answer to this $1 / O$ problem. However, the effectiveness of these techniques recuuires the application to access the data in a sequential manner, or at the very least that the data objects in the file are placed on disks in such a manner as to avoid disk conflicts (akin to bank conflicts in memory systems).

A second impediment to high performance $\mathrm{I} / \mathrm{O}$ is the tile system itself. Contemporary file system interfaces provide no mechanism for indicating how a file will be accessed. Therefore, in order to make performance optimizations, the file system makes assumptions about how the file will be used. But these are frequently wrong.

The ELFS (ExtensibLe File System) that we have beer exploring 1) provides high bandwidth and low latency $1 / O$ to applications programs on high performance architectures, 2) reduces the cognitive burden faced by applications programmers when they attempt to optimize their $\mathrm{I} / \mathrm{O}$ operations to fit existing file system models, and 3) seamlessly manages the proliferation of data formats and architectural differences [GrL91a, GrL91b]. The ELFS solution consists of language and run-time system support that permits the specification of a hierarchy of file classes. Domain specific I/O operations can be specified for each class. High performance is realized by 1) permitting the specification and implementation of caching and prefetching strategies on a class by class basis, 2) using class specific optimization information (such as access pattern specification) passed from the user to the class instance via member functions, 3) permitting ELFS objects to be partitioned across multiple devices in a data and access pattern sensitive fashion, and 4) supportung automatic asynchronous, pipelined, access to file objects and thus permitting the overlap of $\mathrm{I} / \mathrm{O}$ and computation. The cognitive burden on programmers is reduced by encapsulating optimizations in the class specification and by providing high-level, class-specific, operations on tiles, e.g., read matrix by row or column. Finally, data format heterogeneity cain be enciapsulated by ELFS tile objects. 


\subsection{Parallel Stream $\mathbf{I} / \mathrm{O}$}

While ELFS can provide important low-level support at the file level, the way that access commands to it is also crucial. The most commonly used data organization is that of a flat file, or relation, in which a set of data values is represented as a record, or tuple

$$
\left\langle v_{1}, v_{2}, v_{3}, v_{4}, v_{5}\right\rangle
$$

A file format, or relation schema, specifies the order of fields, or attributes, in the record or tuple, together with their characteristics. In ADAMS, this is accomplished by simply enumerating the desired attribute elements. Consequently the following sequence of ADAMS statements is often found in applications programs; in this case they are embedded in a $C$ program.

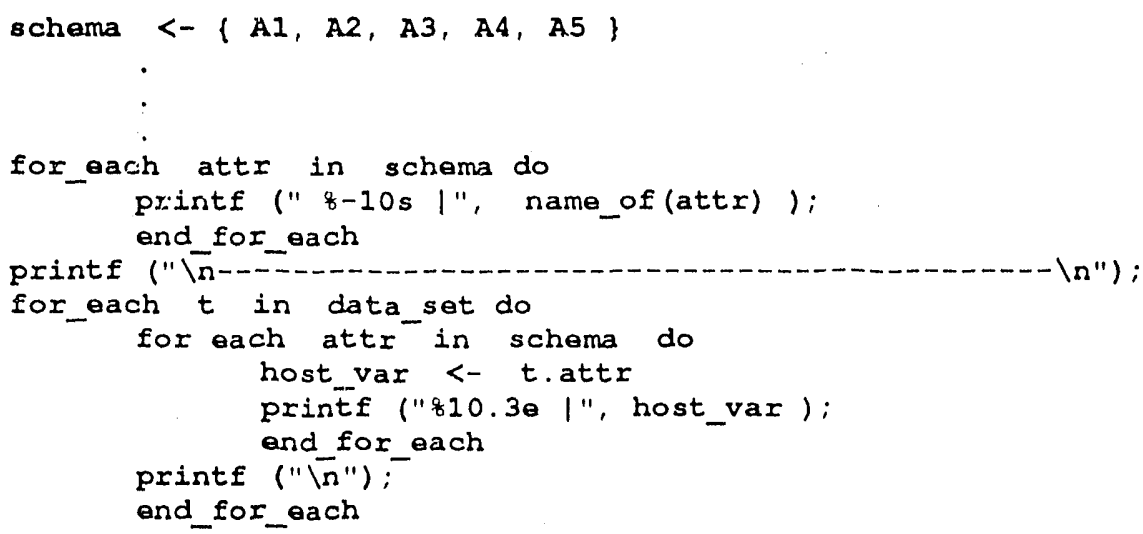

This sequence simply prints the data_set, or relation, in tabular form

\begin{tabular}{|c|c|c|c|c|c|c|c|}
\hline Al & I & $\wedge ?$ & A.3 & 1 & $A A_{1}$ & 1 & $A S$ \\
\hline $8.4630-01$ & ! & $-1.2100-0 !$ & $-6.9130-03$ & $\vdots$ & $7.3000-05$ & & $2.1080+02$ \\
\hline-5.7800 & । & $7.6020-01$ & $-2.6 \% k-$ & : & $1.3000-$ & I & $1.1130+00$ \\
\hline $3.0010-0 ?$ & $i$ & $1.5130-0:$ & $\therefore 3(11-2)$ & : & $\Rightarrow 830(30-0)$ & 1 & 5. $390-01$ \\
\hline . & & . & . & & . & & . \\
\hline . & & $\cdot$ & . & & . & & $\cdot$ \\
\hline$-1.3280-01$ & & $-1.288 .0-0$. & 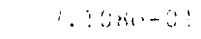 & & $6.5000-05$ & & $3.6920+01$ \\
\hline $4.4 A 5 C-01$ & & $-8.083 .30-01$ & $-3.46 \angle C-0)$ & i & $7.3000-05$ & & $9.2150-02$ \\
\hline
\end{tabular}

It clearly illustrates the repetitive process by which each attribute of a tuple is accessed individually.

Because there are no data dependencies in either of the for each loops, one can establish a parallel stream operation which returns the individual attribute values for all $t$ in data_set in five separate attribute streams. This is schematically illustrated in Figure 2-1. Here, sets and attributes are represented by triangles to indicate their functional nature. Each is enclosed in a manager to suggest their implementation as server objects on separate processors. This is the most basic form of parallel data access, and is the first we have implemented. Implementation and analysis details can be found in [McE91].

After an initial latency (of approximately 13 msecs. in our implementation) to start the streams, each invocation of the ADAMS statement

$$
\text { host_var <- t.attr }
$$

will find the desired t.attr value immediately available. Timing tests on the iPCS/2 hypercube [McE91] have shown that with 4 separate storage managers the inner loop, 


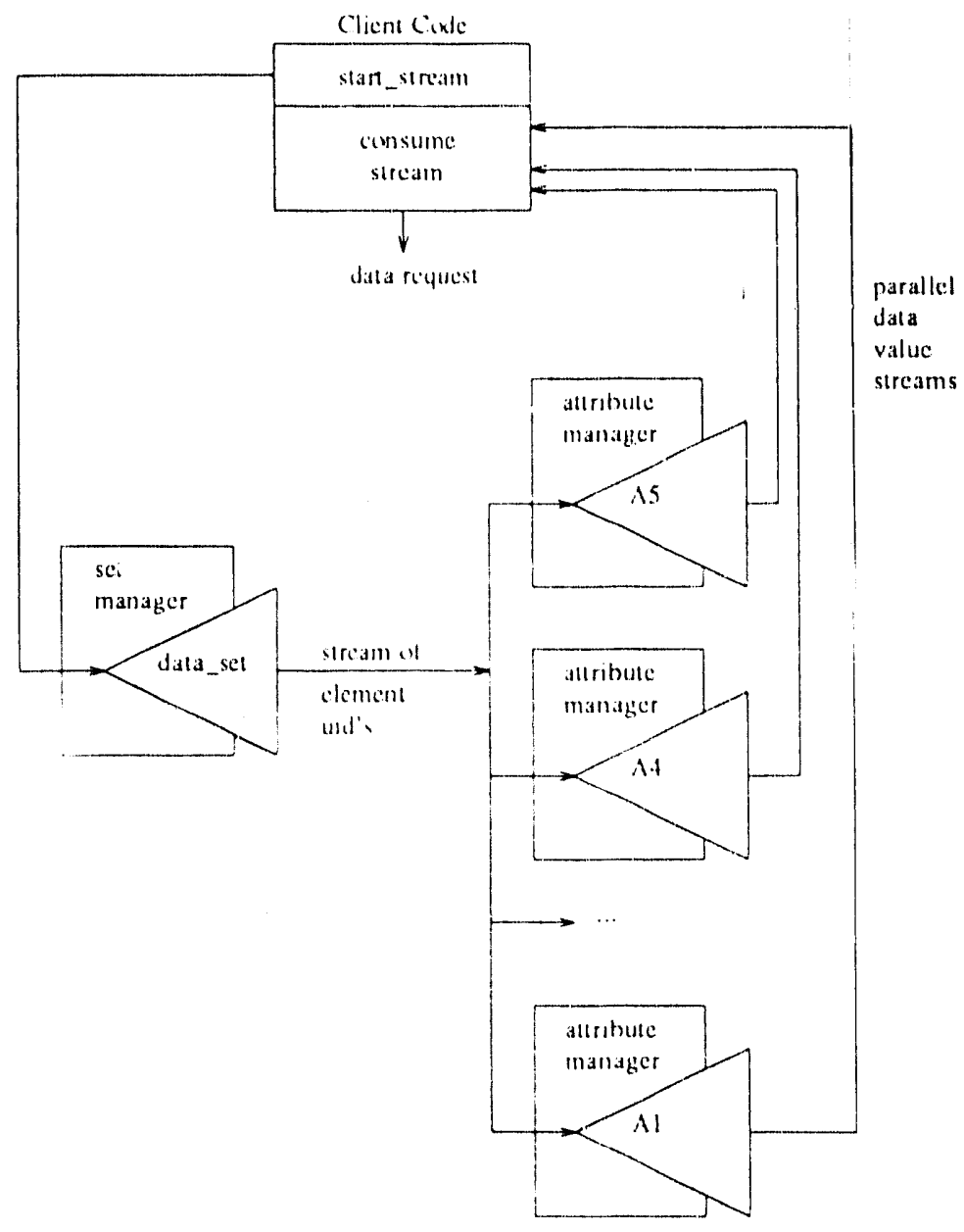

Schematic Representation of Parallel Stream Access

Figure 2-1.

which simply gets all 5 attribute values of a single tuple, iterates at a rate of approximately $5.46 \mathrm{msec}$. per tuple, as shown in tigure 2-2 (solid line). This response time is essentially independent of whether 1 or 6 attribute values are evaluated. From 7 attributes on, the response time climbs until with 12 attributes it is effectively doubled. If 8 storage managers are used (indicated by the dotted line), the access time for 1 to 7 attribute functions remains approximately the same, but the subsequent increase is much less. Tuples with 12 attributes can be delivered to the host program at a rate of one every 7 msecs.

Figure 2-2 illustrates observed performance of our allribute access mechanism schematically shown in Figure 2-1; but one would like to compare it against a theoretical model. Monitoring our system shows that the service time of each storage manager is $S$ $\approx 2.83 \mathrm{msecs}$, where this service time is close to optimal because other studies [FPD91] have shown that the minimum time to deliver a block from the underlying Intel Concurrent File System (CFS) is --2.43 msecs. Lookup by each attribute manager, together with message transfer time, consumes $L=2.14$ msecs. Consequently, the minimal time 


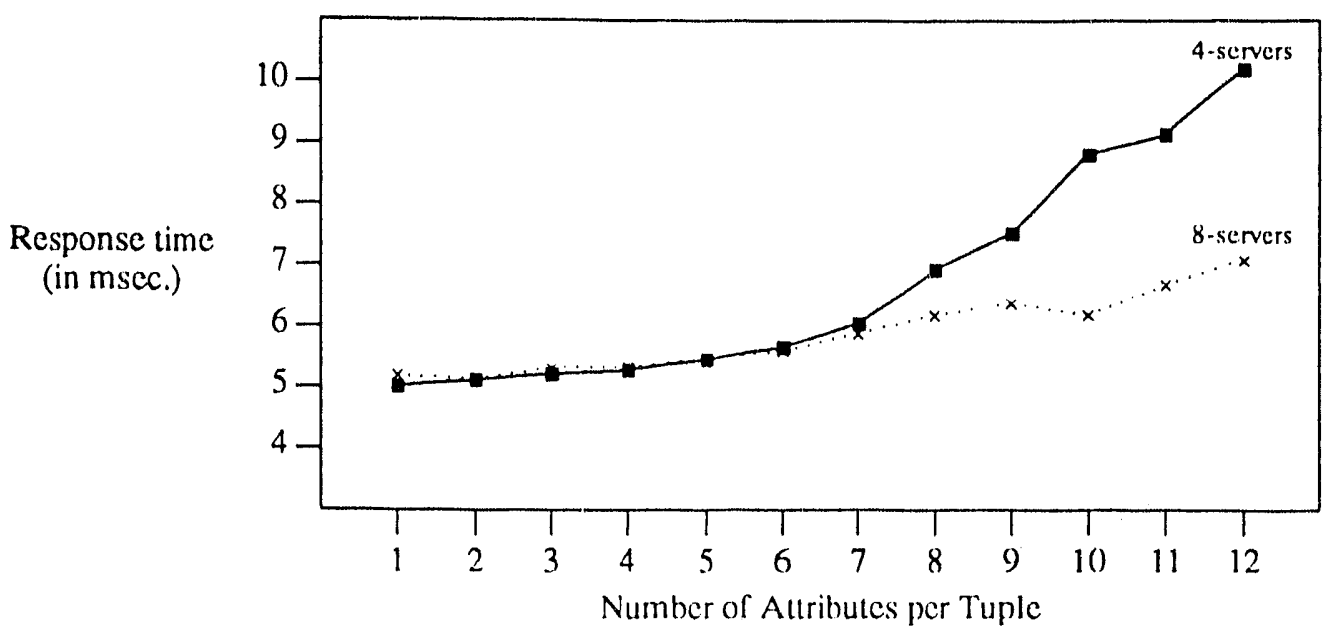

Response Time: msecs. per tuple aceessed

Figure 2-2.

to retrieve an attribute value in our implementation is -4.97 msecs. The real bottleneck occurs when so many attribute servers are making block requests to the storage managers that these req. ests must be queued. Using the operational analysis techniques for queuing systems [BuD78], one can show that queuing at the storage managers must occur when more than 7.02 attributes are being accessed in parallel. At this point the response time is entirely controlled by the average service rate of the $N=4$ storage managers, yielding an asymptotically optimal response time of $S / N \cdot A$, wher : $A$ denotes the number of attribute fuactions accessing storaye in parallel [MCE91]. In Figure 2-3, this

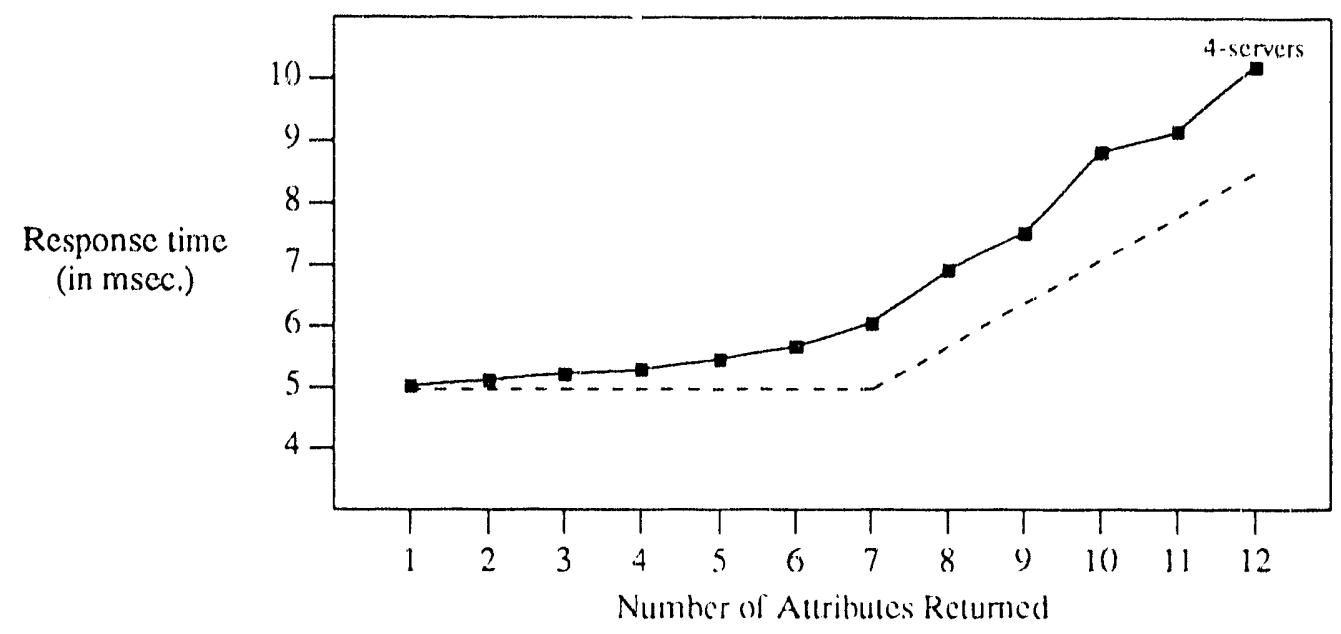

Comparison of Observed Performance with Theoretical Optimal

Figure 2-3. 
asymptotically optimal performance (dashed line) has been superimposed on the observed performance of the system using 4 storage managers. The overall correspondence is apparent. The differences appear to be due to non-uniformity in the distribution of requests to storage managers (uniformity is assumed in the theoretical model) and to message traffic contention (which we were unable to model accurately).

If $N=8$ independent storage managers are available, the same analysis predicts that 14 attribute evaluations can be streamed in parallel before block requests must start being queued. Unfortunately, while we get superior performance with 8 storage managers (as shown in Figure 2-2), we have been unable to empirically verify the predicted behavior, becaus: with only $4 \mathrm{I} / \mathrm{O}$ nodes on our $\mathrm{iPSC} / 2$ disk farm the 8 storage managers cannot be independent.

This mechanism for generating and managing parallel stream $\mathrm{I} / \mathrm{O}$ appears to be quite effective. We wi: be incorporating it into the code that is generated to implement queries, and sequences of data independent ADAMS statements.

\section{Query Processing}

Query processing is often regarded as synonymous with data access. This is not strictly true, because there are many ways of retrieving, or accessing, data besides that of query retrieval. Nevertheless, it is an important way of obtaining datit.

By a query, we mean access to data elements based on specified attribute values, or a combination of such specifications. In an SQL query [KoS86, Mai83], these specifications are treated as the where clause. In formal theory, they are regarded as a predicate with one free variable. ADAMS more closely follows the theoretical approach: Its syntax first defines the concept of a predicate, allowing both existential and universal quantifiers, and then defines a retrieval_set to be

$$
\{\text { <ree_variable> in <restriction_set }>\text { < predicate }>\text { \} }
$$

where the <restriction set>, which may be an arbitrary set expression, ensures that the retrieval is safe, or effectively computable.

One test of the generality of this query approach has been the implementation of a complete SQL front end which parses arbitrary SQL queries (using yacc), generates corresponding ADAMS retrieval expressions, and then executes them [Cle91]. This project clearly illustrates one intended use of ADAMS - as a generic, easily distributed, database manager over which various, more restrictive or more familiar, data interfaces can be constructed.

Implementation of this general query capability was a major effort. It low level indexing techniques employed (0-trees [OrP88, OrP89], which have been described in earlier reports. It nature is best understood by examining a few representative examples. ${ }^{3}$ The following, relatively simple query, retrieves all CS majors in our student - professior - course database (which has been described in a previous report) who have a gpa (grade point average) greater than 3.().

\footnotetext{
${ }^{3}$ All examples have been taken from actual ADAMS programs, coded in C or Fortran, exceuting over a varicty of test data sets.
} 
(1)

$\left\{x\right.$ in undergrad union graduate $\mid x \cdot \operatorname{majox}={ }^{\prime} \mathrm{CS}^{\prime}$ and $\mathrm{x} \cdot \mathrm{gpa}>=\mathbf{\prime}^{\prime} 3.0^{\prime}$ '

The entire expression above denotes a set of elements. It may be assigned to an ADAMS variable, or it may be used in any set expression precisely as any other set designator. Its most notable feature is the use of set union as the restriction set. In the following query, we retrieve all CS graduate students with 3.() or better gpa and whose advisor is named 'Pfaltz'.

(2)

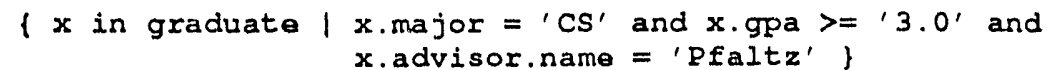

In this query, the ADAMS map advisor implements a rclational join, but much more rapidly. To get the same students, but now any who have a tenured professor as advisor, one would use

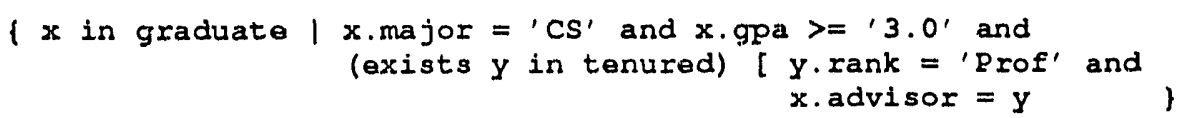

Sorh the existential quantifier and functional maps can be used to replace the rather costly join operator.

Using the Bibiographic Citations Query database, described in the following section, one might use

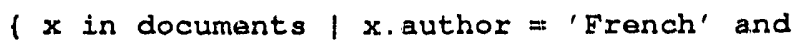

to find any articles written by 'French' that have been included in any book edited by 'Stonebraker'.

Our final example comes from a fairly large database of tactical battlefield data. The query

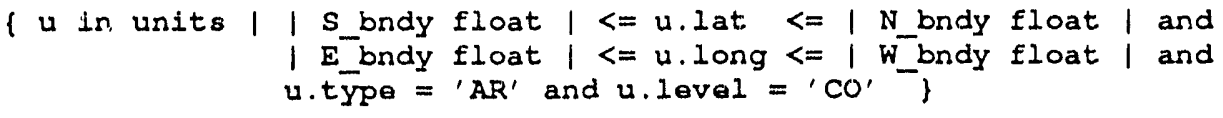

finds all armored companies within a specified geographical region. The spatial range search illustrates how we employ host language variables, e.g. $S$ bndy in ADAMS queries, as well as the more general interval specification syntax which eliminates conjunction on two simple comparators.

The reason for presenting so many examples is to drive home the point that most queries are specified in terms of multiple attributes and/or maps. Because, in (1), major and gpa are distinct attributes, one can retrieve those elements satisfying each condition independently and in parallel using the techniques described in the preceding section. Similarly, in (5), lat, long, type, and level are distinct attributes that can be verified in parallel. One can find elements satisfying all conjuncts as quickly as one can find elements satisfing a single comparative condition. We have hand coded such simple conjunctive queries using the mechanisms described in section 2: however, inclusion of such query optimization in the preprocessor requires substantial data dependence analysis which we have not yet attempted.

Queries (2), (3), and (4) which employ maps that reference other data sets are more complex. We are as yet unsure of the correct way to handle these. Moreover, all queries must eventually require parallel set operations, such as union, intersection, and relative complement. An initial approach to this problem has been described in [PFS891. 
In the course of investigating parallel query implementation, we became aware of a large class of interesting queries that can not be easily handled by any existing retrieval mechanisms. They may be generally categorized as spatial queries. Query (5) is a special case of such spatial queries in which a spatiai rectangle is given as one conjunct. The search is constrained by a bounding rectangle. Many systems can handle this. But consider a query of the form "find all armored companies in: a <specified irregularly shaped region>", or "find all images in a collection of medical irnages containing a dark, convex blob of size greater than 10". We have begun to look at such more general queries, which we discuss more fully in section 6 .

\section{Prototype Implementation}

This project, the development of a "Bibliographic Citations Query System" uses an ADAMS database along with a graphical front-end to provide a tool to users who wish to perform queries on a collection of bibliographic citations. The motivation for the project was two-fold: primarily, to design and implement a real, non-trivial application using ADAMS; and secondarily, to produce a useful tool for retrieval of citations. We wanted to build a real application to gain experience with the ADAMS language and to discover whether there were any significant shortcomings in the langurge. By using a real application we would be forced to overcome any language inadequacies that might be encountered in a satisfactory manner, and not just avoid them.

Building this application using ADAMS serves to (1) determine the adequacy of the language for schema development and data representation, and (2) collect performance measurements by establishing benchmarks for insertion and retrieval of data. The application can also be iised to stress test the ADAMS system by allowing the insertion and querying of a large number of citations. We chose to use the bibliographic citations because we have online access to a very large number of them and because they provide a challenging environment for data representation and retrieval. If the system performs adequately on a non-trivial, computationally intensive application such as this, then it should perform as well or better for applications with simpler data structures. Hence, we can infer more from testing the performance of this application than we might from a simpler one.

Upon insertion into the database, each bibliographic citation causes the creation of at least 3 new sets, 3 maps, and 12 attributes. In addition, there are at least 8 set insertions per new citation. Already, this cne application has uncovered several run-time errors that were invisible with smaller test applications, for example a serious "storage leak" in which storage for temporary sets was not properly released on termination of a run. Even our current data set of approx. 10,000 bibliographic citations generates over 100,000 small, but distinct sets. Since a majority of operations are set operations, we expect this application will be of great use as a stress test of the ADAMS set capabilities. We plan to use it to benchmark future versions of ADAMS, to compare future performance with the current system performance, and to point out features that need to be refined for future versions. 


\section{Synchronization and Reliability: Algorithms and New Paradigms}

We have developed new scheduling algorithms based on the idea of adjusting the serialization order of active transactions dynamically |SoL92|. This is the first successiul attempt to integrate benefits of the pessimistic and optimistic approaches for transaction scheduling. When compared with conventional transaction scheduling algorithms (such as the two-phase locking protocol), our algorithms significantly improve the system performance.

In addition, we have evaluated optimistic concurrency control protocols. Our results indicate that optimistic or hybrid approaches may outperform the pessimistic approach in a wide operational range. Our new algorithms and evaluation results have been presented at the International Conference on Data Engineering [SPL92] and at the International Workshop on Transaction and Query Processing [SLS92].

We have developed priority-ordered deadlock avoidance algorithms and deadlock detection algorithms for distributed resource management $\left|Y_{1}\right| S 92 \mid$. They are basec on partial resource allocation graphs, which provide not only aransaction wait-for information but also resurce allocation information. It combines both path-pushing and edgechasing technique: for efficient resource management.

We also have developed new replication control algorithms. These alyorithms are very efficient for distributed database systems, in which.replicated resources should be managed to support consistency, while providing high performance [SoK92]. Using our prototyping environment, we have implemented those algorithms and demonstrated that they provide higher level of concurrency and greater flexibiiity.

We have developed a new paradigm for multiprocessor database systems, and implemented a parallel programming interface based on our paradigm [CSO91]. Our new paradigm has created new research opportunities for operating systems and databases for parallel computing systems. For example, using the new programming interface, we have developed PRDB, an experimental relational database system that runs on an emulated tightly-coupled, shared-memory multiprocessor system in our prototyping environment. It provides a general paradigm for exploiting parallelism and different scheduling policies. This experimental system has been used for investigating implementation techniques for parallel database systems and the impact of multiprocessor technology on operating systems design.

Fault-tolerant mechanisms and transaction scheduling techniques should be used together to ensure that database systems continue to operate correctly even in the presence of processor failures. We have developed efficient scheduling algorithms for multiprocessor systems and evaluated their performance [Oh91].

We have investigated indexing tree structures for parallel database systems. A variant of the B-tree, called the $B^{*}$ tree is especially well suited for parallel database systems. We have studied the $B^{*}$ tree and its variation, called the $B$-link tree. The B-link tree guarantees that only a constant (small) number of nodes are locked by any update process at any given time. We implemented protocols to manage those tree structures on BBN Butterfly parallel machine for performance evaluation. Our results indicate that the overall performance of the B-link tree protocol is superior thin that of the $B^{*}$ tree protocol. 


\section{Spatial Databases}

One of the important advantages we have claimed for the functional approach of ADAMS is that it simplifies the manipulation of non-traditional data such as images and other spatial data. For this reason we have begun to examine the problems of accessing spatial data, in general, and images, in particular. Moreover, the processing of these usually large data sets is a natural area for parallel processing, and, in fact, many of the Grand Challenge Problems [OOO91] that have driven the High Performance Computing initiative are spatial in nature.

Image access, and other forms of spatial access, clearly require high bandwidth $\mathrm{I} / \mathrm{O}$ performance. But, the major problem is not just that of $\mathrm{I} / \mathrm{O}$. Retrieving the "correct" image is more difficult. Existing query languages and indexing methods just don't seem to work very well. Yet, spatial retrieval naturally occurs in many advanced computer applications, whenever it is beneficial to process objects according to their positions in a D-dimensional space. Traditionally, it has been associated with computer-aided design and geographic applications, but more recently the number of applications that require the spatial-search capability has grown substantially. They include robotics, computer vision, natural-resource management, environmental studies, medical imaging, etc. The types of "spatial queries" that are most useful for these applications require the ability to search efficiently for D-dimensional objects which:

(1) contain a specified point in space (point query);

(2) intersect a specified region in space (region intersection);

(3) enclose a region in space (region enclosure); or

(4) are enclosed by a region in space (region containment) [SeK88].

Many spatial retrieval algorithms assume the simplest spatial representation of objects using minimal enclosing rectangles. As noted in $[\mathrm{SeK} 88 \mid$, they can be organized into three groups: (1) transformation techniques handle spatial queries by mapping each object to a single point in a multi-dimensional space; (2) overlapping-region schemes use hierarchical organization of overlapping rectangles, where each higher-level rectangle encloses several low-level regions; and (3), clipping methods perform decomposition of rectangles along vertical and horizontal lines (or hyperplanes of a higher-dimensional space). Each class of methods appears to have its advantages and disadvantages; but all suffer from imprecision, most spatial objects of interest are not rectangular in nature. More complex vector representations of enclosing polygons with arbitrary sides have been considered, as have pixel-by-pixel representations, which are precise but extremely inefficient in terms of their storage requirements.

Quadtrees and octtrees [Sam84] reduce this overhead by decomposing objects intn constituent squares or cubes, respectively, with variable size; but they too have problems, they are not easy to manipulate. We have been experimenting with a variation of the quadtree concept that makes use of our O-tree indexing mechanism [OrP88], which we call a $\mathrm{Q}_{0}$-tree [OrP91]. It provides the kind of efficient representation that quadtrees do, but compresses them somewhat, while simultaneously allowing dynamic manipulation, such as object insertion, deletion, directly on the tree representation itself. We hope that this method of representing spatial data will eventually lead to more efficient retrieval mechanisms as well. 
Our current research is comparing the $\mathrm{Q}_{0}$-tree approach with the many other spatial representations, such as grid files [NHS84] hB-trees [LoS9)(), R-trees [Gut84] $\mathrm{R}^{+}$-trees [SRF87], k-D-B-trees [Rob81], multi-level grid files [SiW88], and zkd B-trees, [OrM84].

In this report we introduce a dynamic index structure for spatial retrieval, called a $Q_{0}$-tree, which has none of these disadvantages. So far, $Q_{0}$-trees appear to constitute a dynamic index structure for spatial retrieval that avoids the kinds of inefficiencies found in other mechanisms. It provides a fast point search along a single path in the structure, involving only 2 or 3 disk accesses in most realistic situations. It supports arbitrarily shaped objects and search regions, and incurs no false drops, neither with respect to the objects themselves, nor with respect to higher-level blocks within the structure. No entry representing a region that does not overlap the search region will ever be examined to answer a spatial query. If a block of the structure has been accessed, than it contains at least one entry whose corresponding region overlaps the search object. Finally, the index structure itself contains a complete representation of the original image in a much more compact form, and supports various operations on images accurately and efficiently. In that sense, $Q_{0}$-trees are both the spatial structures and the isomorphisms of their corresponding raster images, which eliminate the need to explicitly store the images.

\section{Dynamic Schema Modification and Metadata}

Dynamic schema modification and the representation of metadata are functional capabilities that we have claimed for ADAMS in the past; but we had neither realized their true importance, nor fully tested their implementation until we began reworking the dictionary that implements our shared name space. It turns out that they are extremely important in the development of database systems, in general, and representing scientific data, in particular.

\subsection{Shared Dictionary Development}

Crucial to the implementation of ADAMS, or for that matter any database system, is the existence of a "dictionary" which maintains basic information about its name space, that is about the database itself. In a relational database system, the dictionary will maintain, at least, the names of constituent relations and their schema, because relations and attributes can be named by the user. In a functional database, such as ADAMS, in which classes, sets, attributes, maps, codomains, and instantiated elements can be named, the dictionary is even more important and more complex. Usually, the database dictionary is a utility with its own files and storage that are separate from the database it describes. This is the way that we have implemented ADAMS; its dictionary utility was coded in C with its own file space, while the run-time system is coded in $\mathrm{C}++$ using distributed storage managers. The run-time storage system has been carefully designed and implemented to run in parallel in distributed memory environments. Its data is easily shared. This is not true of our dictionary.

A major goal of the past 6 months has been to implement the dictionary itself as an ADAMS database. This will yield superior performance, because dictionary access can then be performed in parallel, becaluse any improvements to the run-time system will automatically be included in the dictionary utility, and because the dictionary can itself be distributed to avoid having a centralized bottleneck in the system as a whole. 
Moreover, dictionary maintenance will be simplified, because its code will be written in high-level ADAMS statements, rather than $\mathrm{C}$ or $\mathrm{C}++$.

But this is a non-trivial task. One can not define the ADAMS dictionary structure until there is a dictionary to record it!

What is needed is a bootstrap process, with a hand-coded, minimal dictionary defining only the most primitive concepts upon which a more sophisticated dictionary can be constructed. This is best illustrated with an example. A fundamental element in the ADAMS dictionary is an entry of the form

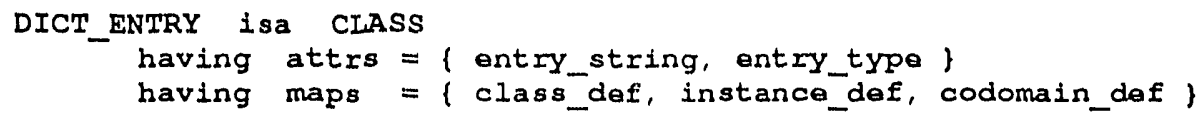

However, an attribute such as entry_string or a map such as class_def can not be defined until we can instantiate a dictionary entry in the class DICT_ENTRY to record its name. Our approach instead has been to make a preliminary DICT_ENTRY class of the form:

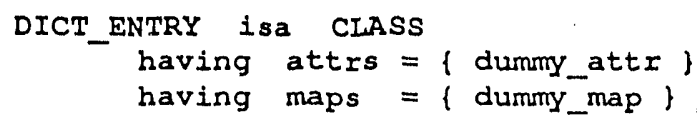

where dummy_attr and dummy_map are primitive string attributes and generic "classless" maps respectively. Once a rudimentary DICT_ENTRY class has been declared, and once an initial entry_string attribute has been instantiated and represented in the dictionary using the initial DICT_ENTRY structure, our existing pre-processor allows us to execute the statements

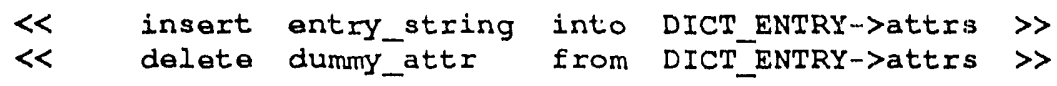

The schema of a DICT_ENTRY has now been dynamically modified to read

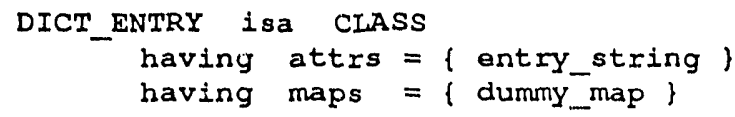

In this way we able to incrementally bootstrap the definition dictionary structures, until the final schema of each is attained.

\subsection{Representation of Scientific Data}

In scientific databases, knowledge based systems, and computer aided design and manufacturing systerns, it is seldom the case that either the procedures that will be used, or their persistent data requirements, can be specified with complete accuracy at the time of design. The latter two technologies presume a kind of indeterminism in which it is expected that the system itself will help the user discover just what data and which configurations are appropriate to the problem at hand. And scientific enquiry is far more than simply collecting data that conforms to a prespecified model. It can be argued, that 
its very essence is just the reverse, that science is the discovery of models, configurations, or structures, which make sense of the observed data at hand. One must create and/or dynamically modify the representational structures in response to observed data or changing scientific goals, that is on-the-fly, in a manner similar to our dictionary modification.

In addition to dynamic modification, the role of metadata in the design and implementation of scientific databases is becoming ircureasingly more important. Everyone recognizes that files, relations, or arrays of data values, such as real numbers, are by themselves of little use. Some additional information, called metadata, is required to interpret the data [FJP90]. Unfortunately, there is no common agreement as to precisely what constitutes metadata. One type of metadata comprises that additional information needed to exchange data. This includes the datum type (e.g. integer, real, string) and the datum length. This metadata, which describes the physical representation of data, is typically encapsulated in the dictionary.

A very different type of metadata comprises that additional information needed to interpret data. This includes, at least, those attribute names occurring in a class, or schema, declaration and which can be extracted from the dictionary. But, one interprets a real number quite differently if it denotes the attribute al than if it denotes the attribute $a 2$. While these attribute names are themselves essential metadata, they are frequently insufficient by themselves to correctly interpret the associated datum in a scientific database. At the very least a researcher reviewing the data would like to know the units of measurement. Note, that normally this is a property of the attribute itself; e.g., we expect that all mass observations will be measured in the same units. Since in a functional approach each attribute has its own identifying uid and can itsolf be treated as a data element, we can consider attributes of attributes. This has always been a feature of the ADAMS syntax, but until now it has been unused. Consider, for example, the definition of a REAL_QUANTITY as

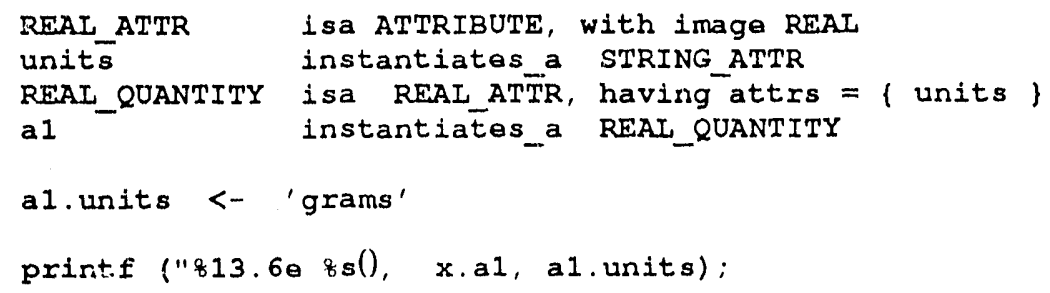

will not only print the current $a 1$ value of the element $x$, but also its units of measurement. If $a 2$ is a linear measurement, it might be instantiated with

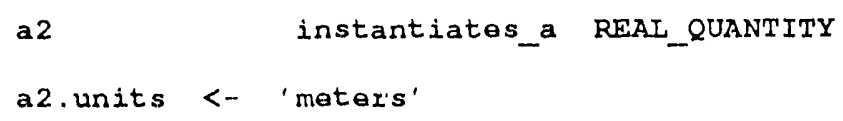

Or, if the precision of the measurement is important, we might dynamically add the real attribute precision to the concept of a REAL_QUANTITY by the sequence 


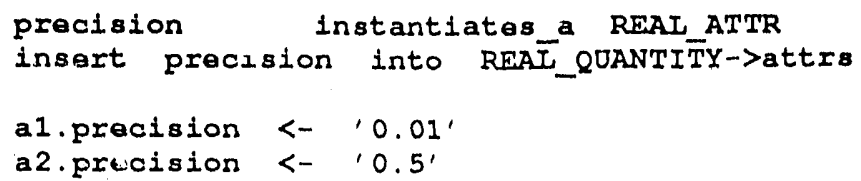

A functional approach to the representation of scientific data does not solve the interpretative metadata problem. But dynamic class modification, attributes of attributes, and attribute class inheritance do provide useful tools by which issues of data interpretation can be more easily attacked. When the ideas above were presented at the Earth and Spaces Sciences Information Systems '92 Conference [PFG92] they were very well received.

\section{Future Research}

Much of the more important research discoveries we have made have been in the context of a testbed environment. This is often sufficient to establish a proof of concept. More importantly, one often needs a carefully crafted testbed in order to be able to accurately monitor performance. However, it is one thing to implement, say parallel stream $\mathrm{I} / \mathrm{O}$, in a hand crafted environment, it is quite another to provide the same parallel performance in user programs. The goal of ADAMS from its inception has always been to create a complete, usable database facility for general computing. Consequently, we must integrate several of our new discoveries into our working system, while at the same time pursuing new avenues of research that will enhance the access to, and use of, scientific data in high-performance computing systems.

More specifically:

(1) We will combine the low-level file access and manipulation capabilities of ELFS with the stream I/O operations descrited in Section 2. Then both must be integrated into the code generator, using source code data dependence analysis. This latter may be hard.

(2) Most synchronization and data concurrency control mechanisms assume serializability as their model of "correct" computation. Even though we have developed scheduling algorithms that minimize the impact of serializability, it can be demonstrated that this model is still unnecessarily restrictive in a parallel environment. In preparation is a graph-theoretic model of synchronization which subsumes both serial and SIMD models as "correct". We will be extending these more general models of synchronization, and then expect to implement them in the ADAMS transaction manager.

(3) We have created several large databases which we used to test the basic capabilities of our ADAMS implementation. But they do not exercise all of its possible constructs fully. We should implement at least two more very large scientific databases.

(4) As more and more scientific databases become involved with spatial data, images, spectral data, and other non-traditional data forms, it becomes important to continue work with these data types. We will continue investigating spatial data representations, as described in Section 6, with particular emphasis on parallel I/O. 
(5) Progress in parallel query algorithms has been disappointing, primarily because we need new algorithms for parallel set manipulation, specifically union, intersection, and relative complement. It will be central on our agenda for the coming year, but without a really significant research breakthrough may be slow. In addition, we will be trying to understand and develop new range search 'capabilities, which assume on!'y partial, rather than total, orders.

(6) The implementation of the dictionary in ADAMS itself should be completed by the end of the summer. Once this is complete, we will create multi-level dictionaries. Also, we intend to explore browsing capabilities based on meta-data and dictionary search. 


\section{References}

[BuD78] J. P. Buzen and P. J. Denning, The Operational Analysis of Queueing Network Models, Computing Surveys 10,3 (Scp. 1978), 255-261.

[Cle91] T. P. Cleary, A Relational Interface to an Object Based System, or Translating SQL to ADAMS, IPC TR-91-009, Institute for Parallel Computation, Univ. of Virginia, Aug. 1991.

[CSO91] R. P. Cook, S. H. Son, H. Y. Oh and J. Lee, New Paradigms for Real-Time Database Systems, 8th IEEE Workshop on Real-Time Operating Systems and Software, Allanta, GA, May 1991, 103-108.

[FJP90] J. C. French, A. Jones and J. L. Pfalt\%, A Summary of the NSF Scientific Database Workshop, IEEE Data Engineering Bulletin 13,3 (Scp. 1990), 55-61.

[FPD91] J. C. French, T. W. Pratl and M. Das, Performance Measurement of a Parallel Inpul/Output System for the Intel iPSC/2 Hypercube, Proc. 1991 ACM Sigmetrics Intern'l Conf. on Measurement and Modeling of Computer Systems, Sin Dicgo, CA, May 1991.

[GPF90] A. S. Grimshaw, J. L. Pfall, J. C. French and S. H. Son, Exploiting Coarse Grained Parallelism in Databasc Applications, PARBASE-90 International Conf. on Databases, Parallel Architectures' and their Applications, Miami Beach, FL. Mar. 1990, 510-512.

[Gri90! A. S. Grimshaw, The Mentat Run-Time System: Support for Medium Grain Parallel Computation, Proc. 5th Distributed Memory Computing Conf., Charleston, SC, Apr. 1990.

[GrL91a] A. S. Grimshaw and E. C. Loyot, ELFS: Object-Oriented Extensible File Systems, Proc. 1991 Parallel and Distributed Information Systems Conf., Miami, FL, Dec. 1991.

[GrL91b] A. S. Grimshaw and E. C. Loyot, ELFS: Object-Oriented Extensible File Systems, Tech. Rep. TR-91-14, Dpt. of Computer Science, Univ. of Virginia, July 1991.

[Gut84] A. Guttman, R-trees: A Dynamic Index Structure for Spatial Scarching, Proc. ACM SIGMOD Conf. on Management of Data, Boston, MA, 1984, 47-57.

[KoS86] H. F. Korth and A. Silberschatz, Database System Concepts, McGraw-Hill, New York, 1986.

[LoS90] D. Lomet and B. Salzberg, The hB-Tree: A Multi-Attribute Access Method with Good Guaranted Performance, Trans. Database Systems 15,4 (Dec. 1990), 625-658.

[Mai83] D. Maier, The Theory of Relational Databases, Computer Science Press, Rockville, MD, 1983.

[McE91] R. McElrath, A Look at Two Persistant Storage Models, IPC TR-91-011, Institute for Parallel Computation, Univ. of Virginia, Dec. 1991.

[NHS84] J. Nicvergelt, H. Hinterberger and K. C. Seveik, The Grid File: An Adaptable, Symmetric Multikcy File Structure, Trans. Database Systems 9,1 (Mar. 1984), 3871.

[OOO91] Grand Challenges: High Performance Computing and Communications, A Report by the Committee on Physical, Malhematical, and Engineering Scientists, Office of Science and Technology Policy, 1991. 
[Oh91] Y. Oh and S. H. Son, Multiprocessor Support for Real-Time Fault-Tolerant Scheduling, IEEE Workshop on Architectural Aspects of Real-Time Systems, San Antonio, TX, Dec. 1991, 76-80.

[OrM84] J. A. Orenstein and T. Merret, A Class of Data Structures for Associative Searchir., Proc. ACM SIGACT News-SIGMOD Conf. Principles Database Sys., Watcrio, Canada, 1984, 181-190.

[OrP88] R. Orlandic and J. L. Pfaltz, Compact 0-Complete Trees, Proc. 14th VLDB Conf., Long Beach, CA, Aug. 1988, 372-381.

[OrP89] R. Orlandic and J. L. Pfaltz, Analysis of Compact 0-Complete Trecs: A New Access Method to Large Databascs, in Proc. 7th FCT Conf., Szeged, Hungary, SpringerVerlag, Berlin-Heiciciberg-New York, Aug. 1989, 362-371.

[OrP91] R. Orlandic and J. L. Pfalti, $Q_{0}$-trecs: A Dynamic Structure for Accessing Spatial Objects with Arbitrary Shapes, IPC TR-91-010, Institute for Parallel Computation, Univ. of Virginia, Dec. 1991.

[PGK88] D. A. Patterson, G. Gibson and R. H. Kalt, A Case for Redundant Arrays of Inexpensive Disks (RAID), Proc. of INTER Conf. on Management of Data, June $1988,108-116$.

[PFS89] J. L. Pfaltz, J. C. French and S. H. Son, Parallel Set Operators, Proc. 4th Conf. on Hypercube Concurrent Computers and Applications, Monterey, CA, Mar. 1989. 481-486.

[PFG91] J. L. Pfaltz, J. C. French and A. Grimshaw, An Introduction to the ADAMS Interface Language: Part I, IPC TR-91-()6, Institute for Parallel Computation, Univ. of Virginia, Apr. 1991.

[PFG92] J. L. Pfaltz, J. C. French, A. S. Grimshaw and R. D. McElrath, Functional Data Representation in Scientific Information Systems, Intern'l Space Year Conf. on Earth and Space Science Information Systems. (ESSIS), Pasadena, CA, Fcb. 1992.

[Pfa92] J. L. Pfalt, A Functional Approach to Scientific Database Implementation, $6^{\text {th }}$ Intern'l Working Conf. on Scientific and Statistical Database Management, Zurich, Switzerland, June 1992, (to appcar).

[PiP89] P.Pierce and J. L. Pfaltu, Evaluation of Complex Line Integrals, Comm. of the ACM 5,2 (June 1962), 345.

[Rob81] J. T. Robinson, The k-D-B-Tree: A Search Structure for Large Multidimansional Dynamic Indexes, Proc. ACM SIGMOD Conf. on Management of Data, Arn Arbor, MI, 1981, 10-18.

[SaG86] K. Salem and H. Garcia-Molina, Disk Striping, International Conf. on Data Engineering, Los Angeles, CA, Feb. 1986, 336-342.

[Sam84] H. Samet, The Quadtrec and Related Hicrarchical Data Structures, Computing Surveys 16,2 (June 1984), 187-260.

[SeK88] B. Seeger and H. Kriegel, Techniques for Design and Implementation of Efficient Spatial Access Methods, Proc: It th VLDB Conf., Long Beach, CA, Aug. 1988, 360371.

[SRF87] T. Sellis, N. Roussopoulos and C. Falout:os, The R+-Tree: A Dynamic Index for Multi-Dimensional Objects, Proc. 13th Conf. on VLDB, Brighton, England, 1987, 507-518.

[SSC91] P. Shebalin, S. H. Son and C. Chang, An Approach to Software Safety Analysis in Distributed Systcms, J. of Computer Systems Science and Engineerin!, 6,2 (Apr. 
1991), 102-116.

[SiW88] H. W. Six and P. Widmaycr, Spatial Searching in Geometric Databascs, Proc. Conf. on Data Engineering, 1988.

[SoC91] S. H. Son and S. Chiang, Evaluation of a Concurrent Checkpointing Algorithm for Distributed Database Systems, International Conference on Parallel and Distributed Computing and Systems, Washington, DC, Oct. 1991, 125-129.

[SoK92] S. H. Son and S. Koloumbis, Replication Control for Distribuled Real-Time Database Systems, 12th International Conference on Distributed Computing Systems, Yokohama, Japan, June 1992, (10 appear).

[SPL92] S. H. Son, S. Park and Y. Lin, An Integrated Real-Time Locking Protocol, Eighth IEEE International Conference on Data Engineering, Phoenix, AZ, Feb. 1992, 527. 534.

[SoL92] S. H. Son and J. Lee, A New Approach to Real-Time Transaction Scheduling, 4th Euromicro Workshop on Real-Time Systems, Athens, Greece, June 1992, (to appear).

[SLS92] S. H. Son, J. Lee and S. Shamsunder, Real-Time Transaction Processing: Pessimistic, Optimistic, and Hybrid Approaches, Second International Workshop on Transactions and Query Processing, Tempe, AZ, Feb. 1992.

[YHS92] Y. Yang, L. Hsu and S. H. Son, Distributed Algorithms for Efficient Deadlock Detection and Resolution, Technical Report Tech. Rep.-92-(06, Dpt. of Computer Science, Univ, of Virginia, Feb. 1992. 


\section{DISTRIBUTION LIST}

1 - 3* Environmental Acquisitions Branch

Procurement and Contracts Division

U. S. Department of Energy

Oak Ridge Operations

Federal Building

200 Administration Road

Oak Ridge, TN $\quad 37831-8758$

Attention: Ms. Barbara J. Jackson, AD-423

Contract Management Branch

4-5 Dr. Thomas A. Kitchens

ER-7 Office of Energy Research

U. S. Department of Energy

Washington, DC 20585

$6-7 \quad$ J. L. Pfaltz

8 J. C. French

9 A. S. Grimshaw

10 S. H. Son

11 A. K. Jones

12-13 E. H. Pancake

** SEAS Postaward Administration

14 SEAS Preaward Administration Files

* Original, unbound and reproducible, plus 2 bound copies.

** Cover letter

$\mathrm{JO \# 4734:ph}$ 

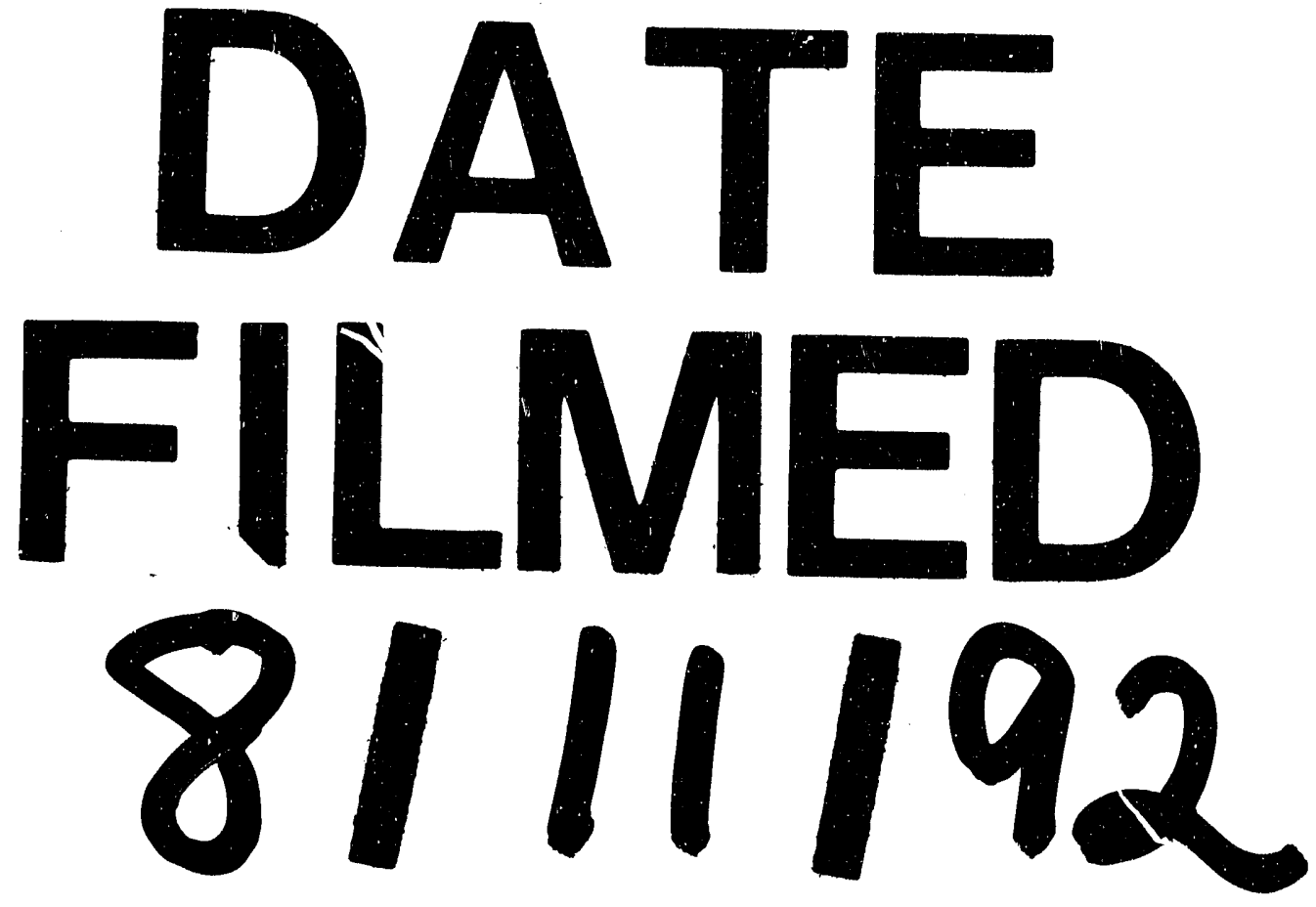

4 
